\title{
New Solutions of Lorentz Transformation III
}

\section{Tomáš Kafoněk}

Studied Technical University in Brno, Faculty of Electrical Engineering ( FE VUT Brno), Czech Republic Email: Kafonek.T@seznam.cz

\author{
Article History \\ Received: January 29, 2021 \\ Revised: February 27, 2021 \\ Accepted: March 5, 2021 \\ Published: March 9, 2021 \\ Copyright (C) 2021 ARPG \& \\ Author \\ This work is licensed under \\ the Creative Commons \\ Attribution International \\ (c) $(5)$ \\ CC BY: Creative \\ Commons Attribution \\ License 4.0
}

\begin{abstract}
This work is my third part of the hypothesis which was originally based on the basic assumptions of the Lorentz transformation and has various consequences. In this first part of the hypothesis [1], I calculated a wave function from the general assumptions of the Lorentz transformation. This wave function describes the spatiotemporal deformations and I use it also in my second part of the hypothesis [2] to calculate the interference and diffraction which resulted in an equation that is not based on complex functions, as is the case with standard calculations. This equation we can examine also, for example, in the context of electron levels in an atom, because interference and diffraction are phenomena related to Young's experiment and the wave properties of electrons have been demonstrated.
\end{abstract}

Keywords: Lorentz transformation; STR; Interference; Diffraction; Atomic orbital.

\section{Introduction}

\section{Energy Levels of a Hydrogen Atom}

The equation for the energy level of an electron around a nucleus can be written as Energy level [3]:

$E_{n}=-h c R_{\infty} \frac{Z^{2}}{n^{2}}$

$\mathbf{R}_{\infty}$ is Rydberg constant, $\mathbf{Z}$ is atomic number, $\mathbf{n}$ is principal quantum number, $\mathbf{h}$ is Planck constant, and $\mathbf{c}$ is speed of light.

For greater accuracy, we use $R_{H}$ for hydrogen instead of $R_{\infty}$ [4] and at the same time we assume that the atomic number $\mathrm{Z}$ is for hydrogen 1 .

So, we assume accordingly:

$E_{H}=-h c R_{H} \frac{1}{n^{2}}$

And we can also write this equation as:

$E_{H}=-h c R_{H} A_{n}$

Where we consider that:

$A_{n}=\frac{1}{n^{2}}$

Now I would like to recall equation (9.19) which I calculated in my second part ofthe hypothesis - in New Solutions of Lorentz Transformation II. Tomáš [2] This equation for energy density will serve our purpose better than the standard equation for calculating intensity from complex numbers, of course,both equations have an almost identical form:

$\left\langle w_{E}\right\rangle+\left\langle w_{M}\right\rangle=\frac{v^{2}}{c^{2}} w_{0} \frac{\sin ^{2} \Psi \cos ^{2} \Phi}{\Psi^{2}}$

We consider a speed comparable to speed $\mathbf{c}$, so we will consider that:

$\lim _{v \rightarrow c} \frac{v^{2}}{c^{2}}=1$

And also, we will considera simpler case in which it is only a matter of diffraction, so:

$\cos ^{2} \Phi=1$

Then we get this simplified equation:

$\left\langle w_{E}\right\rangle+\left\langle w_{M}\right\rangle=w_{0} \frac{\sin ^{2} \Psi}{\Psi^{2}}$

We use the equation (9.13) from Tomáš [2]:

$$
\Psi=\mathrm{k}\left(\frac{\mathrm{d}}{2}\right) \sin \alpha
$$

According to the list of equations in wave theory [5]: 
$\mathrm{k}=\frac{2 \pi}{\lambda}$

We will substitute the equation (10.8) into the equation (9.13):

$\Psi=n_{\Psi} \pi \sin \alpha$

$\boldsymbol{\alpha}$ is a symbol of the angle which is subtended by the direction of radiation and we use this equation:

$\mathrm{n}_{\Psi}=\frac{d}{\lambda}$

$\mathbf{d}$ is a symbol of width of the slit and $\lambda$ is a symbol of the wavelength.

We can get an idea using a physical application. You can use, for example, the application [6]. As we can see, it is the ratio of resonance at the slit.

Now we will transfer this system to the model of the atom, and even in this case we will assume the resonance, so the number $n_{\Psi}$ is a multiple of a wave or half-wave. (10.7):

So,let us look at the course of the maximally simplified function which is based on the form of the equation $\frac{\sin ^{2} \mathrm{x}}{\mathrm{x}^{2}}$

Figure-1.

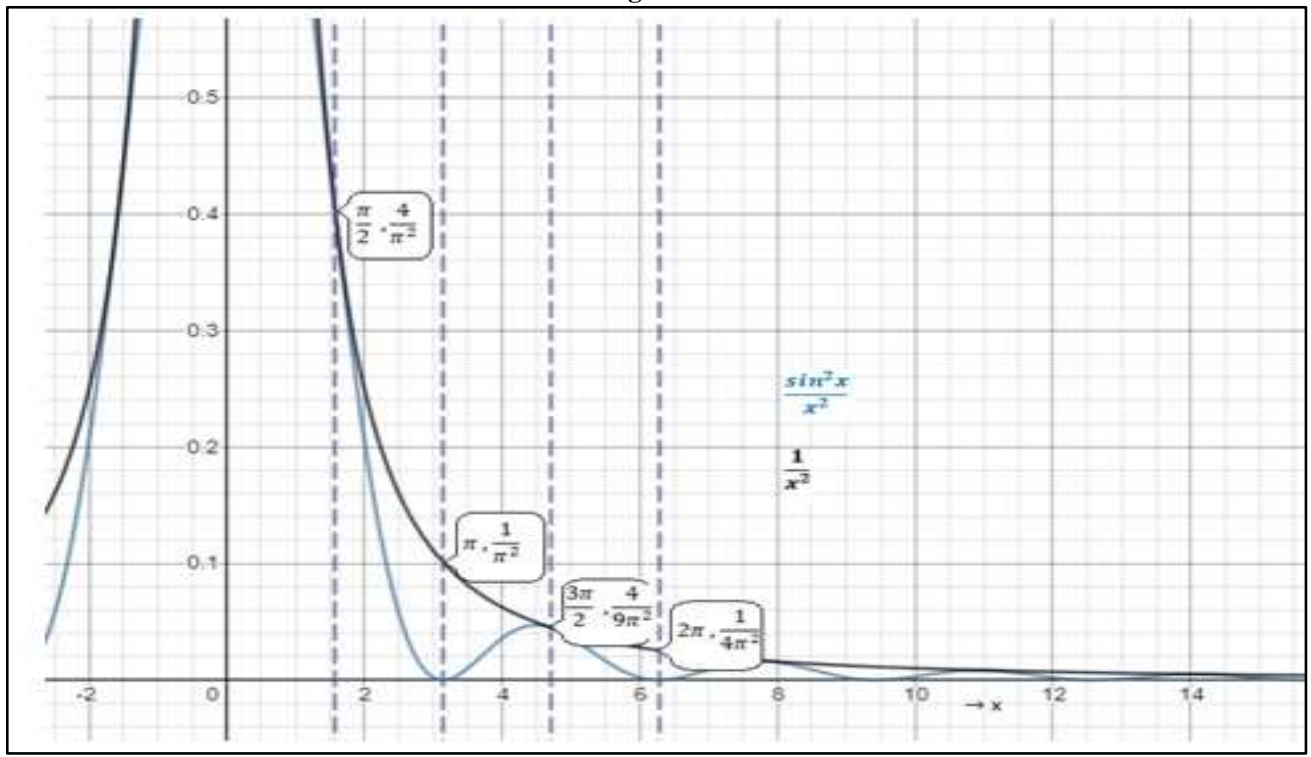

I have found that in a way this function copies the function $\frac{1}{\mathrm{x}^{2}}$.However, this function does not contain the principal quantum number which should fundamentally determine the quantum of energy. So, let us substitute the principal quantum number $\mathbf{n}$ into the denominator of this function. Then we get the following function: $\underline{\sin ^{2} x}$

This function has this graph:

Figure-2.

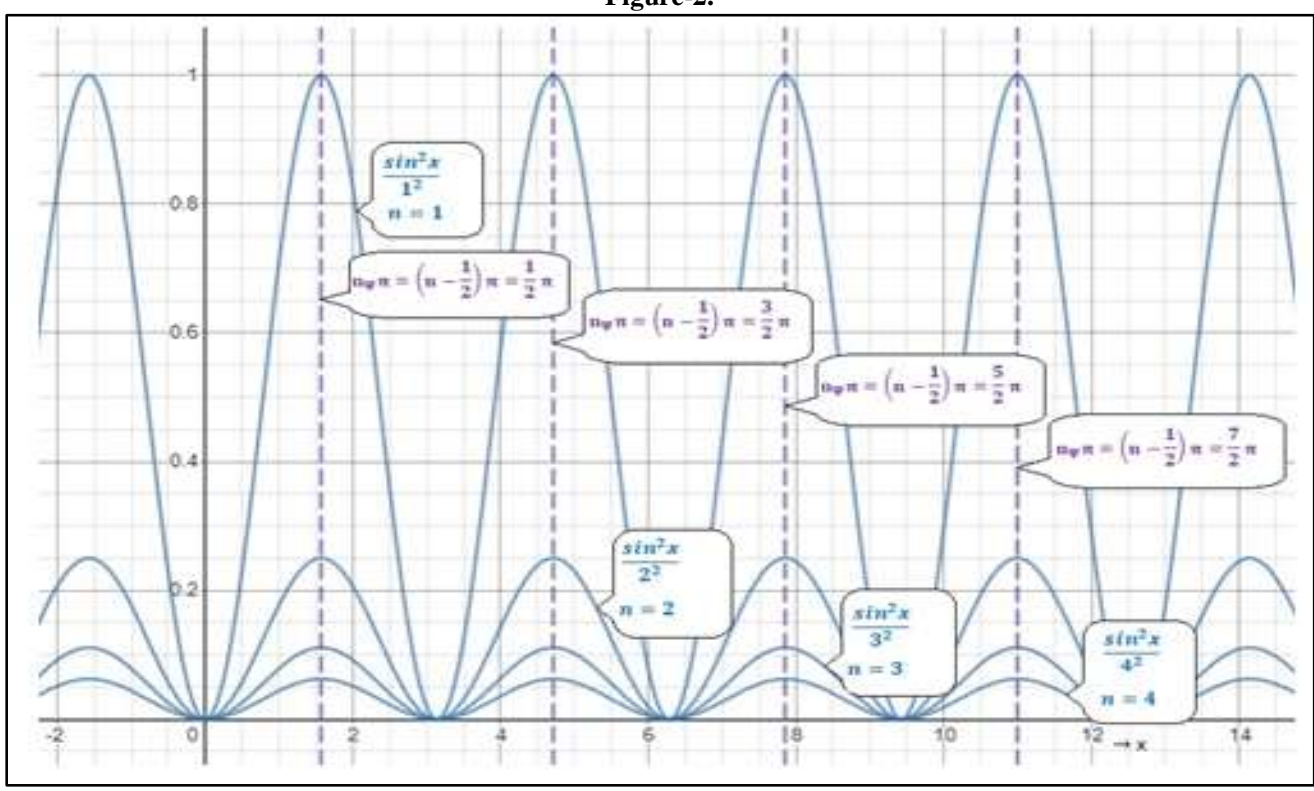


As we can see, maxima of this function lie in the following points: $\pi / 2,3 \pi / 2,5 \pi / 2$ atd. So, if we proceed from the equation (10.4), we will assume that:

$\mathrm{A}_{\mathrm{n}}=\frac{\sin ^{2} \Psi}{\mathrm{n}^{2}}$

We will assume, that exist:

$\mathrm{n}_{\Psi}=\mathrm{n}-\frac{1}{2}$

We will substitute equations (10.9) and (10.12) into the equation (10.11):

$A_{n}=\frac{\sin ^{2}\left(\left(n-\frac{1}{2}\right) \pi \sin \alpha\right)}{n^{2}}$

And, we will substitute the equation (10.13) into the equation (10.3):

$\mathrm{E}_{\mathrm{H}}=-\mathrm{hcR}_{\mathrm{H}} \frac{\sin ^{2}\left(\left(\mathrm{n}-\frac{1}{2}\right) \pi \sin \alpha\right)}{\mathrm{n}^{2}}$

\section{Graph of Distribution of Orbital Energy}

Let us look at the graphs of the equation (10.14) on the next page. These graphs demonstrate a behavior on the layers $13,598 \mathrm{eV}, 3,399 \mathrm{eV}, 1,51 \mathrm{eV}$ etc. It is possible to intuitively recognize which curve of the graph determines which energy level.

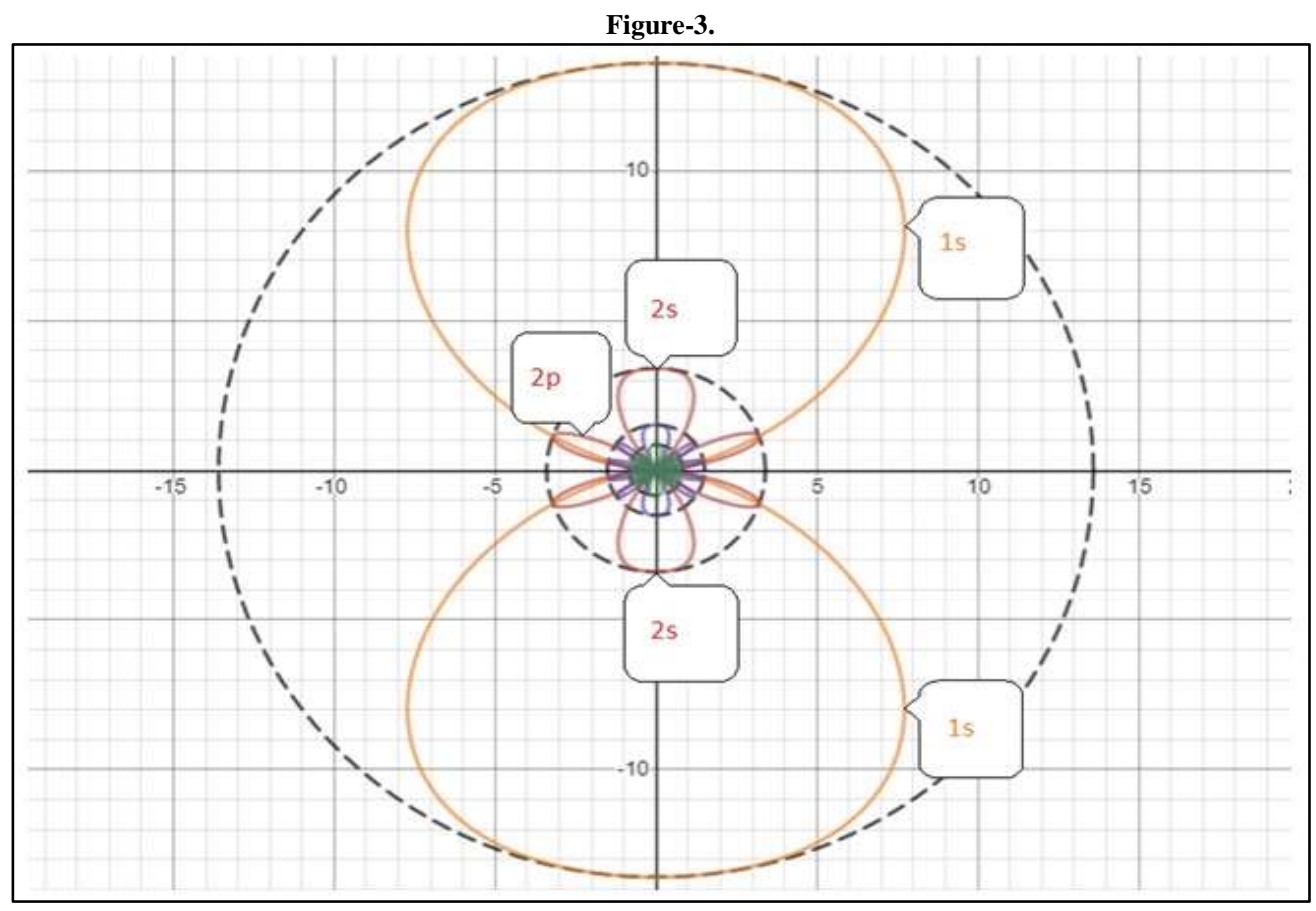

Figure-4.

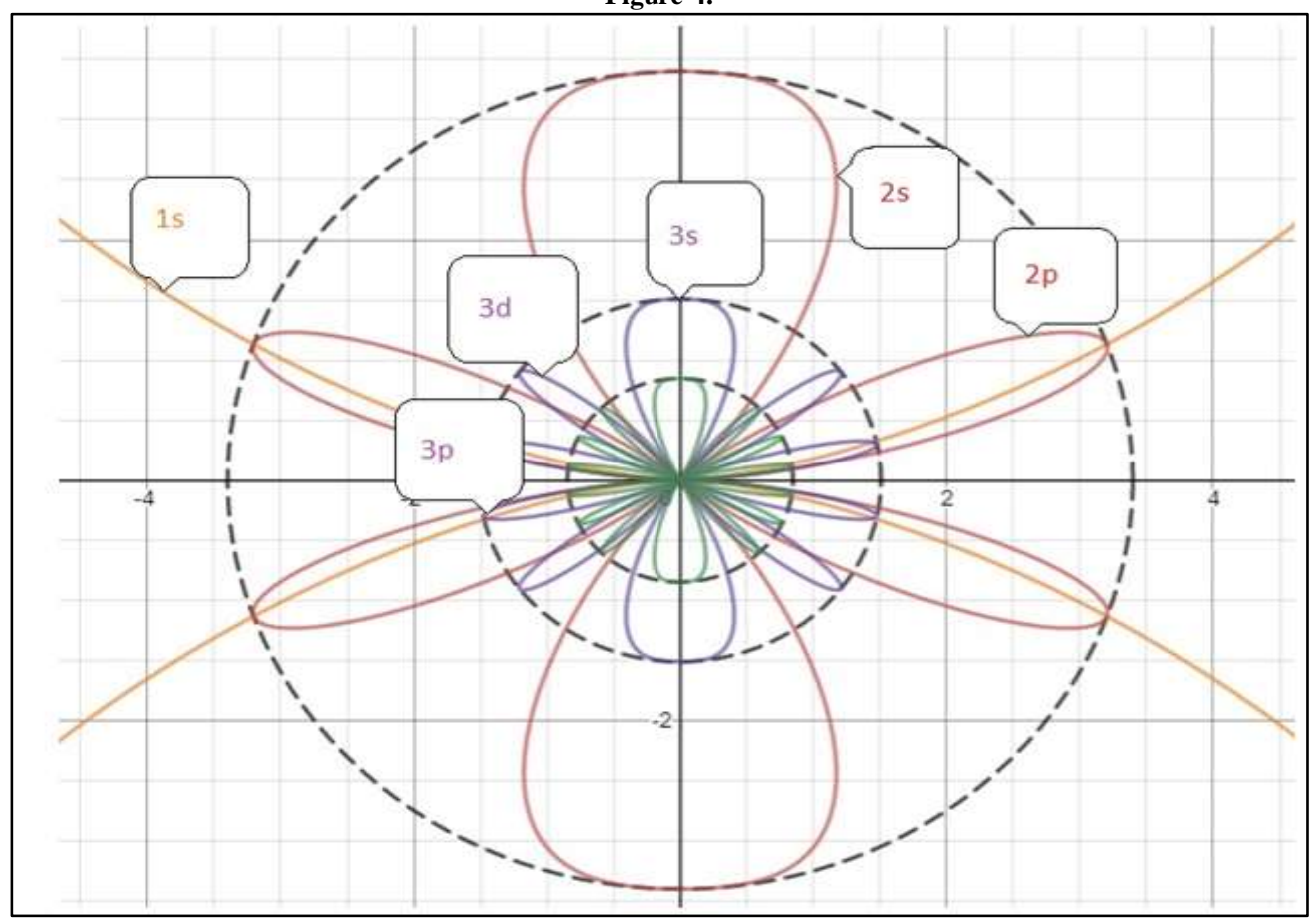




\section{Angle Subtended by Orbit}

If we assume that the shape of the electron orbit does not change even in heavier nuclei, as is generally assumed, we can also use this solution to explain the shape of certain compounds.

We determine the condition of maxima $(n=2)$ and we proceed from the equation $(10.13)$.

$E_{H 2}=-h c R_{H} \frac{\sin ^{2}\left(\left(2-\frac{1}{2}\right) \pi \sin \alpha\right)}{2^{2}}$

That is the second energy level.

So, the following must apply to maxima:

$\sin \left(\left(2-\frac{1}{2}\right) \pi \sin \alpha\right)=\sin \left(\frac{\pi}{2}+\mathrm{k}_{\mathrm{n} 2} \pi\right)$

For our needs we will use the values of $\mathrm{k}_{\mathrm{n} 2}: \mathrm{k}_{\mathrm{n} 2}=0,1,2,$. .

And:

$$
\begin{gathered}
\frac{3}{2} \pi \sin \alpha=\frac{\pi}{2}+\mathrm{k}_{\mathrm{n} 2} \pi \\
\sin \alpha=\frac{1+2 \mathrm{k}_{\mathrm{n} 2}}{3}
\end{gathered}
$$

$\sin \alpha$ must be less than 1 , so this equation makes sense if:

$\sin \alpha_{1}=\frac{1}{3}$

and:

$$
\sin \alpha_{2}=\frac{3}{3}
$$

It will be enough for us to use only these solutions for further calculation. Then we will calculate $\alpha_{2}$ : $\alpha_{2}=\frac{\pi}{2}$

Then we can break down the formula that applies to trigonometric functions:

$\sin \alpha_{1} \sin \alpha_{2}=\frac{1}{2}\left[\cos \left(\alpha_{1}-\alpha_{2}\right)-\cos \left(\alpha_{1}+\alpha_{2}\right)\right]$

We will substitute equations(12.3), (12.4), and (12.5)into the equation (12.6):

We will substitute the equation(12.3):

$$
\begin{gathered}
\frac{1}{3}=\frac{1}{2}\left[\cos \left(\alpha_{1}-\frac{\pi}{2}\right)-\cos \left(\alpha_{1}+\alpha_{2}\right)\right] \\
\frac{1}{3}=\frac{1}{2}\left[\sin \left(\alpha_{1}\right)-\cos \left(\alpha_{1}+\alpha_{2}\right)\right] \\
\frac{2}{3}=\sin \alpha_{1}-\cos \left(\alpha_{1}+\alpha_{2}\right)
\end{gathered}
$$

$\cos \left(\alpha_{1}+\alpha_{2}\right)=-\frac{1}{3}$

$$
\frac{2}{3}=\frac{1}{3}-\cos \left(\alpha_{1}+\alpha_{2}\right)
$$

So, the size of the angle $\left(\alpha_{1}+\alpha_{2}\right)$ isapproximately $109,47^{\circ}$. This value is also stated [7]. The angle $\left(\alpha_{1}+\alpha_{2}\right)$ is a basic angle of platonic solid tetrahedron - for example,the structure of molecule $\mathrm{CH} 4$ istetrahedron.

Figure-5.

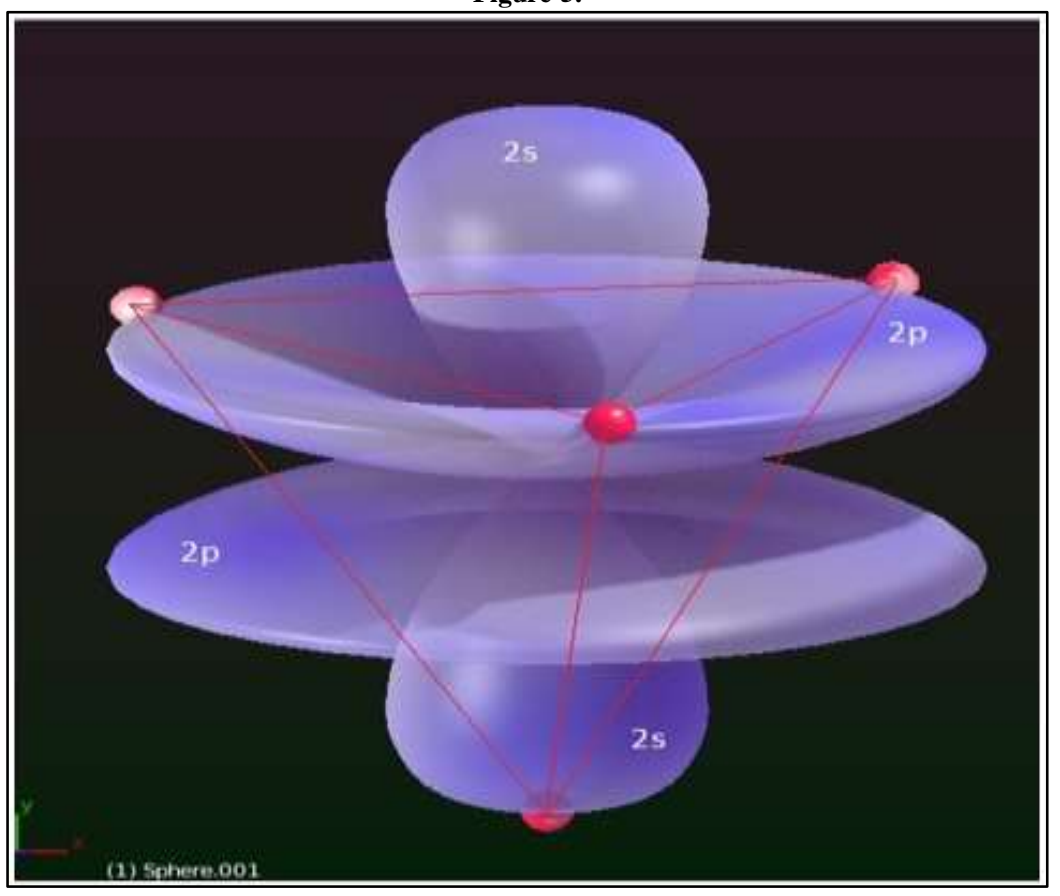




\section{References}

[1] Tomáš, K., 2016. "New solutions of Lorentz transformation." Science Journal of Physics, p. 7. Available: https://www.sjpub.org/sjp/sjp-220.pdf

[2] Tomáš, K., 2020. "New solutions of Lorentz transformation II." EPH - International Journal of Mathematics and Statistics vol. 6, pp. 1-12. Available:

https://ephjournal.org/index.php/ms/article/view/1664/969

[3] Energy level. Available: https://en.wikipedia.org/wiki/Energy_level

[4] Rydberg Constant, 2019. Available: https://en.wikipedia.org/wiki/Rydberg_constant

[5] List of Equations in Wave Theory, 2012. Available:

https://en.wikipedia.org/wiki/List_of_equations_in_wave_theory

[6] Diffraction of Light by a Single Slit, 2003. Available: https://www.walterfendt.de/html5/phen/singleslit_en.htm

[7] Christopher, T. J. "The tetrahedron and 109.5 degrees." Available: http://www.ctralie.com/Teaching/Tetrahedron/ 